Document downloaded from:

http://hdl.handle.net/10251/155019

This paper must be cited as:

Munera-Picazo, S.; Amigo, JM.; Aleixos Borrás, MN.; Talens Oliag, P.; Cubero-García, S.; Blasco Ivars, J. (2018). Potential of VIS-NIR hyperspectral imaging and chemometric methods to identify similar cultivars of nectarine. Food Control. 86:1-10.

https://doi.org/10.1016/j.foodcont.2017.10.037

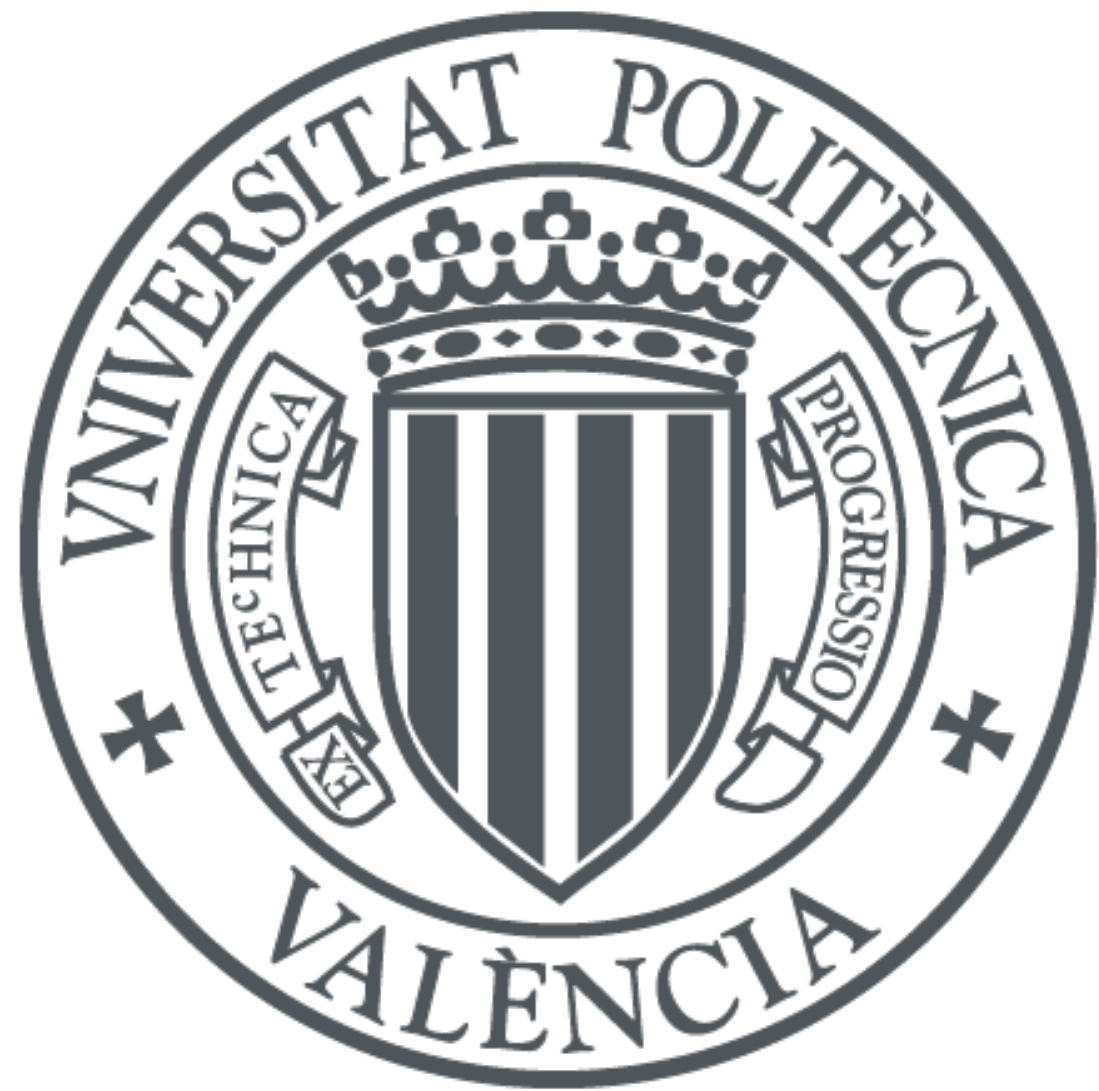

The final publication is available at

https://doi.org/10.1016/j.foodcont.2017.10.037

Copyright Elsevier

Additional Information 


\title{
Potential of VIS-NIR hyperspectral imaging and chemometric methods to identify similar cultivars of nectarine
}

\author{
Sandra Munera ${ }^{\mathrm{a}}$, Jose Manuel Amigo ${ }^{\mathrm{b}, \mathrm{c}}$, Nuria Aleixos ${ }^{\mathrm{d}}$, Pau Talens $^{\mathrm{e}}$, Sergio Cubero $^{\mathrm{a}}$, José \\ Blasco $^{\mathrm{a}^{*}}$ \\ a) Centro de Agroingeniería, Instituto Valenciano de Investigaciones Agrarias (IVIA). Carretera \\ CV-315, Km 10.7, 46113 Moncada, Spain. "blasco_josiva@gva.es \\ b) Department of Food Sciences, Faculty of Science, University of Copenhagen. Rolighedsvej \\ 30, Frederikberg C DK-1958, Denmark. \\ ${ }^{c)}$ Department of Fundamental Chemistry. Federal University of Pernambuco, Av. Prof. Moraes \\ Rego, 1235 - Ciudade Universitária, Recife, Brazil. \\ d) Departamento de Ingeniería Gráfica. Universitat Politècnica de València. Camino de Vera, \\ s/n, 46022 Valencia, Spain. \\ e) Departamento de Tecnología de Alimentos, Universitat Politècnica de València. Camino de \\ Vera, s/n, 46022 Valencia, Spain.
}

\section{ABSTRACT}

Product inspection is essential to ensure good quality and to avoid fraud. New nectarine cultivars with similar external appearance but different physicochemical properties may be mixed in the market, causing confusion and rejection among consumers, and consequently affecting sales and prices. Hyperspectral reflectance imaging in the range of 450-1040 nm was studied as a non-destructive method to differentiate two cultivars of nectarines with a very similar appearance but different taste. Partial least squares discriminant analysis (PLS-DA) was used to develop a prediction model to distinguish intact fruits of the cultivars using pixel-wise and mean spectrum approaches, and then the model was projected onto the complete surface of fruits allowing visual inspection. The results indicated that mean spectrum of the fruit was the most accurate method, a correct discrimination rate of $94 \%$ being achieved. Wavelength selection reduced the dimensionality of the hyperspectral images using the regression coefficients of the PLS-DA model. An accuracy of $96 \%$ was obtained by using 14 optimal wavelengths, whereas colour imaging and a trained inspection panel achieved a rate of correct classification of only $57 \%$ of the fruits. 
Keywords: Stone fruit; quality control; cultivar discrimination; non-destructive; PLS-DA; colour analysis; hyperspectral image.

\section{Abbreviations}

ANOVA $=$ analysis of variance

$\mathrm{CCD}=$ charge-coupled device

$\mathrm{CV}=$ cross validation

$\mathrm{EU}=$ European Union

$\mathrm{F}=$ firmness

$\mathrm{LV}=$ latent variables

$\mathrm{NIR}=$ near infrared

$\mathrm{PC}=$ principal component

$\mathrm{PCA}=$ principal component analysis

PLS-DA = partial least square discriminant analysis

RGB $=$ red, green, blue

$\mathrm{SC}=$ skin colour

$\mathrm{SNV}=$ standard normal variate

$\mathrm{TA}=$ tritratable acidity

TSS $=$ total soluble solids

VIS $=$ visible

\section{INTRODUCTION}

The surface area of the land devoted to the planting of peaches and nectarines (Prunus persica $\mathrm{L}$. Batsch) in the EU was around 232000 ha in 2015/16, with a production of nearly 3.7 million tons of fruit. Spain is the main producer with around 1.4 tons, which accounts for almost $40 \%$ of the total EU peach and nectarine production (USDA, 2016). Due to the importance of nectarine (Prunus persica L. Batsch var. nucipersica) production, it is one of the fruits to which most effort has been devoted by plant breeders in recent years in order to improve agronomic 
performance, and enhanced fruit appearance and quality (Reig, Alegre, Gatius \& Iglesias, 2013 ). This fact has resulted in a significant increase in the number of new cultivars available to fruit growers. These cultivars are similar in appearance but present different sensory properties and therefore different acceptance by the consumer (Iglesias \& Echeverría, 2009). In this context, one of the most widely accepted and cultivated nectarine cultivars in Europe is 'Big Top' due to its presentation, size, sweet taste and low acidity (Echeverría, Cantín, Ortiz, López \& Graell, 2015). However, a stagnation of nectarine consumption is occurring owing to early harvesting, which leads to flavourless fruits being offered with excessive F or irregular quality (Iglesias \& Echeverría, 2009). These authors also point out the lack of an adequate identification of the product in the market. The mixture of sweet and acid cultivars on the shelf could lead to consumer rejection, which in turn might affect sales and prices.

The internal quality assessment of stone fruits has traditionally been performed by destructive methods, which are contaminating, time-consuming and only a few samples per batch can be monitored (Pérez-Marín, Sánchez, Paz, González-Dugo \& Soriano, 2011). Moreover, there is an important lack of classification tools for differentiating cultivars that are very similar to one another. There is therefore a strong need to develop non-destructive and instantaneous methodologies that allow the correct identification of the cultivar in the postharvest stage.

Hyperspectral imaging is a computer vision technique which combines conventional twodimensional digital imagery with spectroscopy to detect spectral features in regions of the electromagnetic spectrum such as the ultraviolet, NIR or infrared regions (Lorente, Aleixos, Gómez-Sanchis, Cubero, García-Navarrete, \& Blasco, 2012). This technique is starting to be used as a scientific tool for quality assurance of a wide range of food including bakery products (Erkinbaev, Henderson, \& Paliwal, 2017; Verdú, Vásquez, Grau, Ivorra, Sanchez \& Barat, 2016), meat (Feng, Makino, Oshita, \& García Martín, 2017; Iqbal, Sun, \& Allen, 2014), or vegetables (López-Maestresalas, Keresztes, Goodarzi, Arazuri, Jaren, \& Saeys, 2016). Fruits are of major interest for the use of this technology in the food industry (Keresztes, Goodarzi \& Saeys, 2016; Munera, Besada, Aleixos, Talens, Salvador, Sun, Cubero, Blasco, \& 2017a). 
However, due to the high importance of other fruits such as citrus or apples, few scientific studies have been done for quality control of stone fruit quality assessment using hyperspectral imaging. Herrero-Langreo, Lunadei, Lleó, Diezma and Ruiz-Altisent (2011) assessed the ripeness of peaches by using multispectral indexes. Lu and Peng (2006) assessed the F of peaches and Zhu, Lin, Nie, Wu and Chen (2016) obtained F distribution maps inside the peach pulp, while Zhang et al. (2015), Li et al. (2016), Pan et al. (2016) and Sun et al. (2017) detected different types of defects and injuries, including decay. Regarding nectarine, Huang et al. (2015) used the same technique to detect defective features and Munera et al. (2017b) to monitor its ripeness.

Hyperspectral imaging generates a huge amount of redundant and frequently highly correlated data that need to be processed (Vélez-Rivera et al., 2014; Sun, Zhang, Liu \& Wang, 2017). To handle such an amount of data and extract the useful information, it must be assisted by chemometric methods. These methods connect chemical measurements with the essential spectral information in order to classify and/or quantify important characteristics. PCA is one of the most popular methods commonly used both to reduce the dimensionality of data and to obtain an overview of all the relevant information in the dataset. It is an unsupervised projection method which summarises data by forming new independent linear combinations of the original variables (Jolliffe, 2002).

PLS-DA is a variant of PLS regression in which the independent variable is categorical, expressing the class membership of the samples. It is performed in order to sharpen the separation between groups of observations by maximising the covariance between the spectra and the independent variable such that a maximum separation among classes is obtained. Furthermore, it is commonly used to understand which variables contain the discriminating information (Lorente et al., 2012). Some examples of the use of this method include the detection of decay lesions in citrus fruits (Folch-Fortuny, Prats-Montalbán, Cubero, Blasco, \& Ferrer, 2016), classification of oat kernels (Serranti, Cesare, Marini, \& Bonifazi, 2013), the classification of edible fennel heads based on the harvest time (Amodio, Capotorto, Chaudhry, 
117 Cho, 2015).

118 In this paper, we put forward a novel approach based on VIS-NIR hyperspectral imaging and chemometric methods to develop statistical predictive models capable of distinguishing cultivars of nectarines with a very similar appearance but different taste. Previous studies have been conducted to differentiate among nectarine cultivars using colour images (Font et al., 2014). However, they use fruits with clearly different appearance. In this work, 'Diamond Ray' and 'Big Top' cultivars have been used due to their similar skin and flesh appearance. Furthermore, these cultivars are grown and marketed at the same time and become a problem for producers when they are mixed, either accidentally or intentionally, in the market.

In addition, using the spectral and spatial information provided by the hyperspectral images, two approaches are further investigated: the first based on the analysis of the individual to establish a practical tool for nectarine classification in the packing houses. order to avoid the further ripening of either cultivar during the experiment.

\section{MATERIAL AND METHODS}

\subsection{Fruit samples}

Nectarines cv. 'Diamond Ray' and 'Big Top' were selected as reference cultivars of sweet and acid cultivars, respectively (Reig, Iglesias \& Echeverría, 2009), due to their similar skin and flesh appearance. These two cultivars are difficult to distinguish by the naked eye, which is problematic for producers when they are mixed in the market.

Fruits were harvested in a commercial orchard in Lerida (Spain) at the commercial maturity stage in the summer season of 2016. A total of 125 fruits of each cultivar without defects or bruises were selected and stored under controlled conditions $\left(1{ }^{\circ} \mathrm{C} ; 90 \%\right.$ relative humidity) in

\subsection{Hyperspectral image acquisition and processing}


The hyperspectral imaging system consisted of an industrial camera (CoolSNAP ES, Photometrics, AZ, USA), coupled to two liquid-crystal tuneable filters (Varispec VIS-07 and NIR-07, Cambridge Research \& Instrumentation, Inc., MA, USA). The camera was configured to acquire images with a size of $1392 \times 1040$ pixels and a spatial resolution of $0.14 \mathrm{~mm} /$ pixel at 60 different wavelengths every $10 \mathrm{~nm}$, in the working spectral range of $450 \mathrm{~nm}-1040 \mathrm{~nm}$. In order to avoid problems of unfocused images due to the refraction of light across this wide spectral range, the focus was adjusted on the central band of the acquisition interval $(740 \mathrm{~nm})$ and the images were captured using lenses capable of covering the whole spectral range without going out of focus (Xenoplan 1.4/23, Schneider Optics, Hauppauge, NY, USA). To optimise the dynamic range of the camera, prevent saturated images and correct the spectral sensitivity of the different elements of the system, a calibration of the integration time of each band was performed by capturing the averaged grey level of a white reference target (Spectralon 99\%, Labsphere, Inc, NH, USA) corresponding to $90 \%$ of the dynamic range of the camera.

The scene was illuminated by indirect light from twelve halogen spotlights (37 W) (Eurostar IR Halogen MR16. Ushio America, Inc., CA, USA) powered by direct current (12 V) and arranged equidistant from each other inside a hemispherical aluminium diffuser. The inner surface of the aluminium diffuser was painted white with a rough texture to maximise its reflectivity, the rough texture being applied in order to minimise directional reflections, which could cause bright spots, thus resulting in highly homogeneous light.

The fruits were introduced manually into a fruit holder, with the stem-calyx axis lying horizontal. Two images of each fruit were acquired using customised software developed at IVIA. A total of 250 images of each cultivar were imported into MATLAB R2015a (The MathWorks, Inc. MA, USA) to be pre-processed using the customised toolbox HYPER-Tools (Amigo, Babamoradia \& Elcoroaristizabal, 2015).

The image processing started with the correction of the relative reflectance by using equation (1) (Gat, 2000):

$$
\rho_{x y}(x, y, \lambda)=\frac{\mathrm{R}^{\text {abs }}}{\mathrm{R}_{\text {white }}^{\text {abs }}}=\rho^{\mathrm{Ref}}(\lambda) \frac{\mathrm{R}(\mathrm{x}, \mathrm{y}, \lambda)-\mathrm{R}_{\text {black }}(\mathrm{x}, \mathrm{y}, \lambda)}{\mathrm{R}_{\text {white }}(\mathrm{x}, \mathrm{y}, \lambda)-\mathrm{R}_{\text {black }}(\mathrm{x}, \mathrm{y}, \lambda)}
$$


where $\rho^{R e f}(\lambda)$ is the standard reflectance of the white reference target (99\% in this work), $R(x, y, \lambda)$ is the reflectance of the fruit captured by the CCD sensor of the camera, $R_{\text {white }}(x, y, \lambda)$ is the reflectance captured by the CCD of the white reference target, and $R_{\text {black }}(x, y, \lambda)$ is the reflectance captured by the CCD while avoiding any light source in order to quantify the electronic noise of the CCD. The images were then clipped and spatially compressed to reduce the computation time, and a proper removal of the background was performed using K-means clustering. Thus, the relative reflectance spectrum of all the pixels in each fruit image was extracted.

\subsection{Colour image acquisition and processing}

Before image acquisition, the SC was analysed to obtain the $L^{*}, a^{*}$ and $b^{*}$ colour coordinates (CIELAB colour space) of each fruit, also using a colorimeter (MINOLTACM700d, Minolta Co. Tokyo, Japan) configured with the standard illuminant D65 and the observer $10^{\circ}$. The SC was obtained as the average of the values of two measurements, one in the blush zone (reddish colour) and another in the ground zone (yellowish colour).

The colour imaging system consisted of a digital camera (EOS 550D, Canon Inc, Japan) arranged inside a square inspection chamber that included a calibrated and uniform illumination system composed of four lamps, each containing two fluorescent tubes BIOLUX 18W/965 (Osram GmbH, Germany) with a colour temperature of $6500 \mathrm{~K}$. The angle between the axis of the lens and the sources of illumination was approximately $45^{\circ}$, and polarising filters were placed in front of the lamps and in the camera lenses to eliminate specular bright spots that could alter the true colour.

The fruits were introduced manually upon a fruit holder, with the stem-calyx axis lying horizontal. Two images were acquired for each fruit, corresponding to each of the two sides delimited by the suture of the fruit. Then, a total of 250 images of each cultivar were imported into customised software developed at IVIA (FoodImage-Inspector v4.0, freely available at http//www.cofilab.com, Spain) to analyse the SC and to obtain the percentage of the reddish and yellowish zones on the fruit. This segmentation was based on the Bayes theorem to assign all 
the pixels in the image to the two classes used in a previous training. The RGB colour coordinates of the acquired images were converted to the $L^{*} a^{*} b^{*}$ coordinates and then corrected using a colour reference target (ColorChecker Digital SG, X-Rite, MI, USA).

\subsection{Visual analysis with trained panel}

The panel was composed of five panellists, ages 29 to 50 years (three male and two female), with expertise in fruit quality and marketing. The panellists were trained using 20 colour images of nectarines of the calibration set (10 from each cultivar chosen at random). A total of 40 colour images of fruits of the validation set (20 from each cultivar chosen at random) were presented with randomised order to each panellist to be classified as belonging to the 'Diamond Ray' or 'Big Top' cultivar.

\subsection{Reference analysis}

The characterisation of the physicochemical properties of the samples using reference methods was performed immediately after the acquisition of the images. F was registered on opposite sides of the fruits using an XT2 Stable texturometer (MicroSystems Haslemere, UK) equipped with a $6 \mathrm{~mm}$ flat plunger. The crosshead speed during the puncture test was $1 \mathrm{~mm} / \mathrm{s}$. The maximum force was expressed in Newton $(\mathrm{N})$. Immediately after SC and F measurements, a juice sample was taken from each fruit for TSS and TA measurements. TSS were determined using a digital refractometer RFM330+VWR (Internacional Eurolab S.L., Barcelona, Spain) at $20{ }^{\circ} \mathrm{C}$ and results were expressed as percentage of TSS. TA was determined using a Crison pHBurette 24 automatic titrator (Crison, Barcelona, Spain) and $\mathrm{NaOH} 0.5 \mathrm{~N}$, according to standard UNE34211:1981 (AENOR, 1981). The results were expressed as the percentage of malic acid. The ANOVA was conducted using the software Statgraphics (Manugistics Corp., Rockville, USA) in order to determine significant differences in the physicochemical properties (F, TSS, TA and $L^{*}, a^{*}$ and $b^{*}$ colour coordinates) between cultivars. 


\subsection{Chemometric methods}

To identify both nectarine cultivars with high precision, two approaches were studied for setting up the classification models: i) including in the model the individual spectrum of each pixel in the nectarine image, and ii) using only the mean spectrum of all the pixels corresponding to each fruit. Thus, 512828 pixel spectra were used in the first approach, and the mean spectra of 500 fruits were used for the second. The data of all the fruits of both cultivars were collected and randomly partitioned into two sets: two thirds of the samples were used to calibrate the models (calibration set) and for cross-validation, while the remaining third was used for independent test prediction (validation set).

Both the directly acquired spectrum of each pixel and that obtained as an average for each fruit were pre-processed using SNV in order to reduce the physical variability between samples due to light scatter (Rinnan et al., 2009). This correction was performed using the equation (2):

$$
x_{\text {corr }}=\frac{x_{\text {org }}-a_{0}}{a_{1}}
$$

Where $\mathrm{x}_{\text {corr }}$ and $\mathrm{x}_{\text {org }}$ are the corrected and raw spectra, respectively, $a_{0}$ is the average value of the sample spectrum to be corrected and $a_{l}$ is the standard deviation of the sample spectrum.

Later, mean centring was applied to normalise the full spectrum. Multivariate analyses were then performed using the PLS_Toolbox (Eigenvector Research Inc., USA) and the HYPERTools toolbox (Amigo et al., 2015) both working under MATLAB R2015a.

PCA was used to explore the differences between the two cultivars using the pixel and mean spectra of the calibration set previously pre-processed by means of SNV and mean centring. Later, PLS-DA models were built to sort the fruits into one of the two studied cultivars. The models were also calibrated using the pre-processed pixel and mean spectra of the calibration set and tested using only samples of the validation or prediction set.

In order to compare the performance of the hyperspectral imaging with the colour imaging system, a PLS model was also built using the mean value of the $L^{*} a * b^{*}$ colour coordinates. 
252 to obtain an estimation of the error rate of the models. All models were statistically validated by using the sensitivity, specificity, class error and accuracy (Eq. 3, 4, 5 and 6):

$$
\text { Sensitivity }=\frac{T P}{T P+F N}
$$

where TP and TN stand for true positive and true negative, respectively, accounting for the samples that have been correctly assigned as belonging (TP), or not belonging (TN), to a specific class. FP and FN stand for false positive and false negative, respectively, accounting for the samples that have been wrongly assigned as belonging (FP), or not belonging (FN), to a specific class.

The ANOVA, using the software Statgraphics, was also conducted in order to determine significant differences in the accuracy of the models.

\section{RESULTS AND DISCUSSION}

\subsection{Cultivar characterisation}

\subsubsection{Physicochemical properties}

Table 1 shows the results obtained from the reference analysis of the physicochemical properties. $\mathrm{F}$ is one of the physicochemical properties commonly used to assess ripeness. In this work, the measures of $\mathrm{F}$ obtained for both cultivars showed no statistical differences, which means that they were in a similar stage of ripeness. According to the mean value of $\mathrm{F}$ measured for each cultivar, these fruits were considered as being within the group that Valero, Crisosto and Slaughter (2007) described as 'ready to buy'.

As noted above, the principal difference between these two cultivars is the flavour; i.e. the typical TSS values for 'Big Top' being higher than in 'Diamond Ray' and vice versa for TA. 
Ray' had $0.8 \%$ TA and $10.3 \%$ TSS. The difference in TSS content between these cultivars may

be attributable to the stage of maturity, the season or the production area (Crisosto, 1994).

Table 1. Results of analysis of physicochemical properties of both cultivars of nectarine

\begin{tabular}{|c|c|c|c|c|c|}
\hline \multirow{2}{*}{\multicolumn{2}{|c|}{ Property }} & \multicolumn{2}{|c|}{ 'Diamond Ray' } & \multicolumn{2}{|c|}{ 'Big Top' } \\
\hline & & Mean & SD & Mean & SD \\
\hline \multirow{2}{*}{\multicolumn{2}{|c|}{$\begin{array}{c}\text { Firmness }(\mathrm{N}) \\
\text { Total soluble solids }(\%)\end{array}$}} & $33.8^{\mathrm{a}}$ & 9.5 & $34.8^{\mathrm{a}}$ & 7.1 \\
\hline & & $11.9^{\mathrm{b}}$ & 1.6 & $12.7^{\mathrm{a}}$ & 2.3 \\
\hline \multicolumn{2}{|c|}{ Tritratable acidity $(\%)$} & $0.7^{\mathrm{a}}$ & 0.1 & $0.4^{\mathrm{b}}$ & 0.1 \\
\hline \multirow{3}{*}{$\begin{array}{l}\text { Skin colour by } \\
\text { colorimeter }\end{array}$} & $L^{*}$ & $36.9^{a}$ & 6.6 & $36.5^{\mathrm{a}}$ & 6.0 \\
\hline & $a^{*}$ & $27.0^{\mathrm{a}}$ & 4.2 & $26.2^{\mathrm{a}}$ & 3.9 \\
\hline & $b^{*}$ & $13.3^{\mathrm{a}}$ & 5.1 & $13.4^{\mathrm{a}}$ & 4.9 \\
\hline \multirow{5}{*}{$\begin{array}{l}\text { Skin colour by } \\
\text { imaging }\end{array}$} & $L^{*}$ & $28.0^{\mathrm{a}}$ & 8.6 & $27.0^{\mathrm{a}}$ & 8.2 \\
\hline & $a^{*}$ & $44.9^{\mathrm{a}}$ & 5.4 & $41.0^{\mathrm{b}}$ & 5.5 \\
\hline & $b^{*}$ & $27.2^{\mathrm{a}}$ & 8.8 & $24.8^{\mathrm{b}}$ & 8.6 \\
\hline & Blush zone $(\%)$ & $67.0^{\mathrm{a}}$ & 21.4 & $66.3^{\mathrm{a}}$ & 18.4 \\
\hline & Ground zone (\%) & $33.0^{\mathrm{a}}$ & 21.4 & $33.7^{\mathrm{a}}$ & 18.4 \\
\hline
\end{tabular}

Different superscript letters in the same row indicate significant differences between cultivars ( $p$ value $<0.05) . S D=$ standard deviation;

The mean $L^{*}, a^{*}$, and $b^{*}$ colour coordinates of the SC using the colorimeter were not statistically different between cultivars (Table 1). However, colorimeters measure small regions only, which can be a major limitation in applications where distinguishing the colours all over the sample is of interest. This means that they are not well suited to measuring objects with a heterogeneous colour (Gardner, 2007), such as nectarines of these cultivars. However, a colour camera provides images in which the colours of the pixels are determined individually (Cubero, Aleixos, Moltó, Gómez-Sanchis \& Blasco, 2011), along with their spatial distribution. The analysis of the colour of the nectarines using imaging enable the evaluation of the SC of the different colour zones separately and calculation of the relative distribution (percentage) of reddish or yellowish colour in the whole fruit. 
Using this percentage, a mean value of the $L^{*}, a^{*}, b^{*}$ coordinates was calculated from the

images. On average, a reddish colour was present on $67 \%$ of the fruit surface and a yellowish colour on $33 \%$ in both cultivars (Table 1). Even so, the mean colour using imaging indicated that the $a^{*}$ and $b^{*}$ scores were statistically different in the two cultivars, i.e. both were higher in 'Diamond Ray'. However, the differences were too small to be detected visually by the human eye, especially during a rapid fruit-sorting process.

\subsubsection{Spectral analysis}

Differences between cultivars were observed in their hyperspectral spectra (Fig. 1). The preprocessed (SNV) mean spectra of the two cultivars followed a similar spectral pattern but had clear differences at specific wavelengths.

In the VIS region, no apparent differences could be visualised in the range between 400$600 \mathrm{~nm}$ where carotenoids are present. In contrast, the 'Big Top' cultivar had lower reflectance (higher absorbance) than 'Diamond Ray' near $680 \mathrm{~nm}$, which is associated with chlorophylls (Lleó et al., 2011; Rajkumar, Wang, Elmasry, Raghavan \& Gariepy, 2012), suggesting a higher content of this molecule. This agrees with the differences in the values of $a^{*}$ and $b^{*}$ found in the colour analysis (Table 1).

In the NIR region, the absorption bands for acids and sugars are usually found around 800 $\mathrm{nm}$ and $840 \mathrm{~nm}$ respectively, attributable to the hydroxyl groups of these compounds (Malegori et al., 2017; Yang, Sun, Pu, Wang \& Zhu, 2015). However, only small differences are usually observable due to the water absorption bands which dominate the spectrum (Nicolaï et al., 2007). In this region, the main differences observed in the spectra were at wavelengths above $850 \mathrm{~nm}$ and, in particular, around $970 \mathrm{~nm}$, where Lu \& Peng (2006) described a peak associated with water absorption, which in this case was more pronounced in 'Big Top' nectarines (Fig. 1). 


\subsection{Overview of the spectral data}

A PCA was performed in order to obtain an overview of the distribution of the spectral data information from the samples of both cultivars. The PCA results from the individual pixel spectra and the mean spectra of each fruit are shown in Figures 2 and 3, respectively.

Forty samples of each cultivar were randomly selected to provide individual pixel spectra and this data was used to generate a score image plot. The first two PCs explained $87.8 \%$ of the total variance $(76.5 \%$ and $11.3 \%$, respectively). The variations in the colour within each fruit showed the distribution or content of the biochemical constituents. A possible trend was discerned in PC2, where pixels with low values (dark blue) were found mostly in 'Big Top' samples; however, there was little difference in individual fruit spectra of the 'Diamond Ray' and 'Big Top' cultivars.

In the PCA of the mean spectra of the calibration set, the first two PCs (Fig. 3A) explained $93.3 \%$ of the variance ( $81.4 \%$ and $11.9 \%$, respectively). The ellipses for the two cultivars appeared distinct, but discrimination between them was not possible because of overlap (Fig. $3 \mathrm{~A})$.

Although the loadings obtained for PC1 and PC2 (Fig. 3B) might have offered information on the most important wavelengths to distinguish the cultivars, this was not useful because separation was not evident in the preceding plot (Fig. 3A). PCA maximises the variance in the first components, which may or may not be related to the segregation of the classes; this does not guarantee the class separability of data due to its unsupervised nature (Jolliffe, 2002).

\subsection{Cultivar classification using individual pixel spectrum}

A PLS-DA model was performed using the spectral range of $450-1040 \mathrm{~nm}$ and the spectrum of the individual pixels of each fruit of the calibration set. The values obtained for sensitivity and specificity (Table 2) indicated that the number of samples correctly identified as belonging to a specific cultivar, or not, was above 0.80 in the CV set, using five LV. Sensitivity 
of 0.83 and 0.86 was determined for 'Diamond Ray' and 'Big Top' respectively being the accuracy of classification $84.8 \%$ and error 0.15 .

Using the spatial data collected by the imaging system the combined results were applied to the calibration set. The predicted class of each pixel was obtained by introducing the spectrum measured for those pixels into the previously built model, and visualising the result. Each pixel was coloured blue if it was assigned to 'Diamond Ray' or red if it was assigned to 'Big Top', as shown in Figure 4A. The accuracy of this classification was $83.8 \%$ and error 0.16 .

To classify each fruit using this approach, the whole fruit was assigned to the class found in the majority of its pixels (Fig. 4B). In this case, the accuracy and the classification error were $84.4 \%$ and 0.16 . In both cases, 'Big Top' was also the best discriminated, with a sensitivity of about 0.90 .

360

Table 2. Cultivar discrimination using the pixel spectrum approach

\begin{tabular}{|c|c|c|c|c|c|c|c|}
\hline$\# \mathrm{~V}$ & \#LV & Set & Class & Sensitivity & Specificity & Error & Accuracy $(\%)$ \\
\hline \multirow{8}{*}{60} & \multirow{8}{*}{5} & & 'DR' & 0.83 & 0.86 & \multirow{2}{*}{0.15} & \multirow{2}{*}{84.8} \\
\hline & & Calibration & 'BT' & 0.86 & 0.83 & & \\
\hline & & Cross & 'DR' & 0.83 & 0.86 & \multirow{2}{*}{0.15} & \multirow{2}{*}{84.8} \\
\hline & & Validation & 'BT' & 0.86 & 0.83 & & \\
\hline & & Validation & 'DR' & 0.79 & 0.89 & \multirow{2}{*}{0.16} & \multirow{2}{*}{83.8} \\
\hline & & pixel & 'BT' & 0.89 & 0.79 & & \\
\hline & & Validation & 'DR' & 0.78 & 0.91 & \multirow{2}{*}{0.16} & \multirow{2}{*}{84.4} \\
\hline & & object & 'BT' & 0.91 & 0.78 & & \\
\hline
\end{tabular}

\subsection{Cultivar classification using mean fruit spectrum}

The sensitivity and specificity in the results of calibration using the mean fruit spectrum approach giving values above 0.90 using six LV (Table 3). In this case, both cultivars were discriminated similarly and the accuracy of classification of the CV was $93.2 \%$ and error 0.07 .

In order to get a graphical view of the veracity of the classification obtained using the validation set, the class for each fruit was predicted by introducing the mean spectrum measured into the previously built model. The result was visualised showing the fruit coloured blue if the 
mean value was assigned by the model to 'Diamond Ray' or red if it was assigned to 'Big Top' (Fig. 5A). The results for the validation set were similar to those obtained in the calibration, showing an accuracy of $94.4 \%$ with a classification error of 0.06 . The ANOVA results indicated that the mean spectra model was significantly better than the pixel model $(\mathrm{p}<0.05)$ to classify the fruits

As Williams and Kucheryavskiy (2016) pointed out, using properly computed object features as the mean spectrum decreases the amount of data, leading to more stable classification models. Furthermore, this approach avoids classifying by pixels when objects from different classes contain many similar pixels and are easily miss-assigned to the opposite class, such as for the cultivars studied in this work. On the other hand, it is important to include the negative influence of the sphericity of the fruits on the reflectance of the light. As seen in Figure 4A, most errors occur at the borders of the fruit, since the centres are usually well illuminated. The pixels near the borders are therefore more likely to be wrongly classified, thus affecting the overall result. In contrast when using the mean fruit spectrum, the averaging minimises these errors.

Table 3. Cultivar discrimination using the mean spectrum approach

\begin{tabular}{|c|c|c|c|c|c|c|c|}
\hline$\# \mathrm{~V}$ & \#LV & Set & Class & Sensitivity & Specificity & Error & Accuracy (\%) \\
\hline \multirow{6}{*}{60} & \multirow{6}{*}{6} & \multirow{2}{*}{ Calibration } & 'DR' & 0.94 & 0.94 & \multirow{2}{*}{0.06} & \multirow{2}{*}{93.8} \\
\hline & & & 'BT' & 0.94 & 0.94 & & \\
\hline & & Cross & 'DR' & 0.93 & 0.94 & \multirow{2}{*}{0.07} & \multirow{2}{*}{93.2} \\
\hline & & Validation & 'BT' & 0.94 & 0.93 & & \\
\hline & & \multirow{2}{*}{ Validation } & 'DR' & 0.94 & 0.94 & \multirow{2}{*}{0.06} & \multirow{2}{*}{94.4} \\
\hline & & & 'BT' & 0.94 & 0.94 & & \\
\hline
\end{tabular}

\subsection{Selection of the optimal wavelengths}

In order to optimise the algorithms for an automatic in-line sorting system working at high speed, it is important to reduce the computational complexity generated by the huge amount of data obtained by hyperspectral imaging systems. This problem is commonly alleviated by techniques that retain the information in the few bands that reveal the most variability and therefore most significant information in the hyperspectral image (Du \& Sun, 2006). The 
method used in this study was the vector of the regression coefficients. This measures the association between each variable and the response and selects variables in two steps: (i) the PLS-DA model is fitted to the data, and (ii) the variable selection is based on a threshold (Mehmood, Liland, Snipen \& Sæbø, 2012). Variables with a high absolute value can be selected because they make the highest contribution to cultivar classification and those with a small absolute value can be ignored. In this study, the regression coefficients were obtained from the PLS-DA model using the mean fruit spectrum approach, due to its higher accuracy in the classification of both cultivars.

Figure 6 shows the vector of regression coefficients. Those peaks where the absolute value was highest were selected as important wavelengths. In the VIS region the selected wavelengths were at 630, 650, 680 and $720 \mathrm{~nm}$ while in the NIR region they were 750-770, 790, 810-840, 860 and $900 \mathrm{~nm}$.

The optimised PLS-DA model was performed using the 14 selected wavelengths as input. The sensitivities and specificities in the CV were similar to the full model using six LV (Table 4). In the prediction set, using only the 14 wavelengths, the sensitivity for the two cultivars increased from 0.94 for both to 0.95 and 0.98 , in 'Diamond Ray' and 'Big Top' respectively. Figure 5 shows the results of both classifications, using the full spectrum (Fig. 5A) and the optimal wavelengths (Fig. 5B) in which more fruits were coloured as they should be when the wavelengths selected as the most important. However, the accuracy obtained, 96.3\%, was not statistically different $(\mathrm{p}>0.05)$ from the accuracy of the full model $(96.3$ and $94.4 \%$, respectively)

Table 4. Cultivar discrimination using the mean spectrum and the optimal wavelengths methods

\begin{tabular}{|c|c|c|c|c|c|c|c|}
\hline$\# \mathrm{~V}$ & \#LV & Set & Class & Sensitivity & Specificity & Error & Accuracy (\%) \\
\hline \multirow{4}{*}{14} & \multirow{4}{*}{6} & Calibration & $\begin{array}{l}\text { 'DR' } \\
\text { 'RT' }\end{array}$ & $\begin{array}{l}0.94 \\
0.94\end{array}$ & 0.94 & 0.06 & 93.8 \\
\hline & & \multirow{2}{*}{$\begin{array}{c}\text { Cross } \\
\text { Validation }\end{array}$} & 'DR' & $\begin{array}{l}0.94 \\
0.93\end{array}$ & $\frac{0.94}{0.94}$ & \multirow[b]{2}{*}{0.07} & \multirow[b]{2}{*}{93.2} \\
\hline & & & 'BT' & 0.94 & 0.93 & & \\
\hline & & Validation & $\begin{array}{l}\text { 'DR' } \\
\text { 'BT' }\end{array}$ & $\begin{array}{l}0.95 \\
0.98\end{array}$ & $\begin{array}{l}0.98 \\
0.95\end{array}$ & 0.04 & 96.3 \\
\hline
\end{tabular}




\subsection{Hyperspectral imaging vs. colour and visual analysis}

When the validation set was classified visually by the trained panel, the same fraction of each cultivar was identified correctly (Table 5). However, the accuracy was very low, i.e. 54.5\% with a classification error of 0.46 . This demonstrates difficulty of the human eye to distinguish between the similar external appearances of these cultivars.

Classification by the colour data had similar accuracy ( $\mathrm{p}$-value $>0.05$ ) to that achieved by the trained panel (Table 5), i.e. 56.9\% accuracy and error of 0.43 . This is especially poor in comparison with the results of the hyperspectral imaging using 14 wavelengths, i.e. $96.3 \%$, error 0.04 (Table 4).

These results are in agreement with the work carried out by Nogales-Bueno, RodríguezPulido, Heredia and Hernández-Hierro (2015) that used NIR hyperspectral and colour imaging to discriminate between four red grape cultivars. Only $52 \%$ of the samples were correctly classified using colour imaging but this figure increased to $86 \%$ using hyperspectral imaging. Furthermore, Font et al. (2014) described an in-line system for verification of nectarine cultivars with close harvest times using different colour space layers of the SC histogram. The success of their technique was $100 \%$ in comparing fruits of three cultivars with a single cultivar for reference. In the same experiments, human classification achieved $86 \%$ accuracy, likely attributable to the large differences in the SC of the cultivars tested. the NIR region. 
Table 5. Cultivar discrimination using colour imaging and by a trained panel

\begin{tabular}{|c|c|c|c|c|c|c|c|c|}
\hline & $\# \mathrm{~V}$ & \#LV & Set & Class & Sensitivity & Specificity & Error & $\begin{array}{c}\text { Accuracy } \\
(\%)\end{array}$ \\
\hline \multirow{3}{*}{$\begin{array}{c}\text { Colour } \\
\text { imaging } \\
\text { (PLS- } \\
\text { DA) }\end{array}$} & \multirow{3}{*}{3} & \multirow{3}{*}{2} & Calibration & $\begin{array}{l}\text { 'DR' } \\
\text { 'BT' }\end{array}$ & $\begin{array}{l}0.75 \\
0.61 \\
\end{array}$ & $\begin{array}{l}0.61 \\
0.75\end{array}$ & 0.32 & 68.0 \\
\hline & & & $\begin{array}{c}\text { Cross } \\
\text { Validation } \\
\end{array}$ & $\begin{array}{l}\text { 'DR' } \\
\text { 'BT' }\end{array}$ & $\begin{array}{l}0.75 \\
0.62 \\
\end{array}$ & $\begin{array}{l}0.62 \\
0.75 \\
\end{array}$ & 0.32 & 68.3 \\
\hline & & & Validation & $\begin{array}{l}\text { 'DR' } \\
\text { 'BT' }\end{array}$ & $\begin{array}{l}0.65 \\
0.49\end{array}$ & $\begin{array}{l}0.49 \\
0.65\end{array}$ & 0.43 & 56.9 \\
\hline $\begin{array}{c}\text { Trained } \\
\text { panel }\end{array}$ & - & - & Validation & $\begin{array}{l}\text { 'DR' } \\
\text { 'BT' }\end{array}$ & $\begin{array}{l}0.54 \\
0.55\end{array}$ & $\begin{array}{l}0.55 \\
0.54\end{array}$ & 0.46 & 54.5 \\
\hline
\end{tabular}

452

\section{CONCLUSIONS}

The capability of VIS-NIR hyperspectral imaging to discriminate very similar cultivars of nectarine has been demonstrated in this work.

The classification of these two cultivars by colour imaging or by a trained panel was very poor, achieving an accuracy of only 56.9\% and 54.5\% respectively. However, hyperspectral imaging supported by chemometric methods and optimised by reduction of the spectral and spatial information enabled classification more accurately than by traditional manual or colourbased systems, and it is also faster than destructive methods.

The use of the mean spectrum of the fruit as input of the predictive models provided classification accuracy of $94.4 \%$. To cope with the huge amount of data captured by the hyperspectral systems, the vector of the regression coefficients of a PLS-DA model identified 14 wavelengths which were selected as optimal, producing the best classification model with a classification accuracy of $96.3 \%$.

This technique may have potential as a tool for rapid and non-destructive cultivar discrimination, allowing the selection of fruit that is better suited to the consumer's preferences. Nevertheless, the results of this study should be confirmed on a larger sample set of fruits grown in different areas and harvested at different stages of ripeness before they can be implemented in an in-line system. 


\section{ACKNOWLEDGEMENTS}

This work was partially funded by INIA and FEDER funds through project RTA201500078-00-00. Sandra Munera thanks INIA for the FPI-INIA grant num. 43 (CPR2014-0082), partially supported by European Union FSE funds. The authors wish to thank Fruits de Ponent (Lleida) for providing the fruit.

\section{REFERENCES}

AENOR. (1981). Productos derivados de frutas y verduras, determinación de la acidez valorable. UNE 34211: 1981

Amigo, J.M., Babamoradia, H. \& Elcoroaristizabal, S. (2015). Hyperspectral image analysis. A tutorial. Analytica Chimica Acta 896, 34-51.

Amodio, M.L., Capotorto, I., Chaudhry, M.M.A. \& Colelli, G. (2017). The use of hyperspectral imaging to predict the distribution of internal constituents and to classify edible fennel heads based on the harvest time. Computers and Electronics in Agriculture 134, 1-10

Crisosto, C.H. (1994). Stone fruit maturity indices: a descriptive. Postharvest News and Information 6, 65-68.

Cubero, S., Aleixos, N., Moltó, E., Gómez-Sanchis, J. \& Blasco, J. (2011). Advances in Machine Vision Applications for Automatic Inspection and Quality Evaluation of Fruits and Vegetables. Food Bioprocess Technology 4, 487-504

Du, C.J. \& Sun, D.W. (2006). Learning techniques used in computer vision for food quality evaluation: a review. Journal of Food Engineering, 72, 39-55.

Echeverría, G., Cantín, C.M., Ortiz, A., López, M.L. \& Graell, J. (2015).The impact of maturity, storage temperature and storage duration on sensory quality and consumer satisfaction of 'Big Top' nectarines. Scientia Horticulturae 190, 179-186.

Erkinbaev, C., Henderson, K., \& Paliwal, J. (2017). Discrimination of gluten-free oats from contaminants using near infrared hyperspectral imaging technique, Food Control 80, 197203. 
Feng, C.H., Makino, Y., Oshita, S., \& García-Martín J.F. (2017). Hyperspectral Imaging and Multispectral Imaging as the Novel Techniques for Detecting Defects in Raw and Processed Meat Products: Current State-of-the-Art Research Advances. Food Control, In press, DOI: /10.1016/j.foodcont.2017.07.013.

Folch-Fortuny, A., Prats-Montalbán, J.M., Cubero, S., Blasco, J. \& Ferrer, A. (2016). VIS/NIR hyperspectral imaging and N-way PLS-DA models for detection of decay lesions in citrus fruits. Chemometrics and Intelligent Laboratory Systems 156, 241-248

Font, D., Tresanchez, M., Pallejà, T., Teixidó, M., Martinez, D., Moreno, J. \& Palacín, J. 2014. An image processing method for in-line nectarine variety verification based on the comparison of skin feature histogram vectors. Computers and Electronics in Agriculture $102,112-119$.

Gardner, J.L. (2007). Comparison of calibration methods for tristimulus colorimeters. Journal of Research of the National Institute of Standards and Technology 112, 129-138.

Gat, N. (2000). Imaging spectroscopy using tunable filters: A review. Technical report, OptoKnowledge Systems Inc. OKSI.

Giné-Bordonaba, J., Cantina, C.M., Larrigaudière, C., López, L., López, R. \& Echeverría, G. (2014). Suitability of nectarine cultivars for minimal processing: The role of genotype, harvest season and maturity at harvest on quality and sensory attributes. Postharvest Biology and Technology 93, 49-60.

Herrero-Langreo, A., Lunadei, L., Lleó L., Diezma, B. \& Ruiz-Altisent, M. (2011). Multispectral Vision for Monitoring Peach Ripeness. Journal of Food Science 2, 178-187.

Huang, F., Zhang, S., Yang, Y., Man, Z., Zhang, X. \& Wu, Y. (2015). Application of hyperspectral imaging for detection of defective features in nectarine fruit. Transactions of the Chinese Society for Agricultural Machinery 11, 252-259.

Iglesias, I. \& Echeverría, G. (2009). Differential effect of cultivar and harvest date on nectarine colour, quality and consumer acceptance. Scientia Horticulturae 120, 41-50. 
Iqbal A., Sun, D.-W., \& Allen, P. (2014). An overview on principle, techniques and application of hyperspectral imaging with special reference to ham quality evaluation and control. Food Control 46, 242-254.

Jolliffe, I.T. (2002). Principal component analysis (2nd ed.). New York: Springer.

Kandpal, L. M., Lee, S. , Kim, M.S., Bae, H. \& Cho, B.K. (2015). Short wave infrared (SWIR) hyperspectral imaging technique for examination of aflatoxin B1 (AFB1) on corn kernel. Food Control 51, 171-176.

Keresztes, J.C., Goodarzi, M., \& Saeys, W. (2016). Real-time pixel based early apple bruise detection using short wave infrared hyperspectral imaging in combination with calibration and glare correction techniques. Food Control 66, 215-226.

Li, J., Chen, L., Huang, W., Wang, O., Zhang, B., Tian, X., Fan, S. \& Li, B. (2016). Multispectral detection of skin defects of bi-colored peaches based on VIS-NIR hyperspectral imaging. Postharvest Biology and Technology 112, 121-133.

López-Maestresalas, A., Keresztes, J.C., Goodarzi, M., Arazuri, S., Jaren, C., \& Saeys W. (2016). Non-destructive detection of blackspot in potatoes by Vis-NIR and SWIR hyperspectral imaging. Food Control 70, 229-241.

Lorente, D., Aleixos, N., Gómez-Sanchis, J., Cubero, S., García-Navarrete, O. L. \& Blasco, J. (2012). Recent advances and applications of hyperspectral imaging for fruit and vegetable quality assessment. Food Bioprocess Technology 5, 1121-1142.

Lu, R. \& Peng, Y. (2006). Hyperspectral scattering for assessing peach fruit firmness. Biosystems Engineering 93, 161-171.

Malegori, C., Nascimento, E. J., Tonetto de Freitas, S., Pimentel, M. F. \& Casiraghi, E. (2017). Comparing the analytical performances of Micro-NIR and FT-NIR spectrometers in the evaluation of acerola fruit quality, using PLS and SVM regression algorithms. Talanta, 165, $112-116$

Mehmood, T., Liland, K. H., Snipen, L. \& Sæbø, S. (2012). A review of variable selection methods in Partial Least Squares Regression. Chemometrics and Intelligent Laboratory Systems 118, 62-69. 
Munera, S., Amigo, J.M., Blasco, J., Cubero, S., Talens, P. \& Aleixos, N. (2017a). Ripeness monitoring of two cultivars of nectarine using VIS-NIR hyperspectral reflectance imaging. Journal of Food Engineering 214, 29-39.

Munera, S., Besada, C., Aleixos, N., Talens, P., Salvador, A., Sun, D.-W., Cubero, C. \& Blasco, J. (2017b). Non-destructive assessment of the internal quality of intact persimmon using colour and VIS/NIR hyperspectral imaging. LWT - Food Science and Technology 77C, 241248.

Nicolaï, B.M., Beullens, K., Bobelyn, E., Peirs, A., Saeys,W., Theron, K.I., Lammertyn, J., 2007. Nondestructive measurement of fruit and vegetable quality by means of NIR spectroscopy: a review. Postharvest Biology and Technology 46, 99-118.

Nogales-Bueno, J., Rodríguez-Pulido, F. J., Heredia, F. J. \& Hernández-Hierro J. M. (2015). Comparative study on the use of anthocyanin profile, color image analysis and near-infrared hyperspectral imaging as tools to discriminate between four autochthonous red grape cultivars from La Rioja (Spain). Talanta 131, 412-416.

Pan, L., Zhang, Q., Zhang, W., Sun, Y., Hua, P. \& Tu, K. (2016). Detection of cold injury in peaches by hyperspectral reflectance imaging and artificial neural network. Food Chemistry $192,134-141$.

Pérez-Marín, D., Sánchez, M. T., Paz, P., González-Dugo, V. \& Soriano, M. A. (2011). Postharvest shelf-life discrimination of nectarines produced under different irrigation strategies using NIR-spectroscopy. LWT - Food Science and Technology 44, 1405-1414.

Rajkumar, P., Wang, N., EImasry, G., Raghavan, G.S.V. \& Gariepy, Y. (2012). Studies on banana fruit quality and maturity stages using hyperspectral imaging. Journal of Food Engineering 108, 194-200.

Reig, G., Alegre, S., Gatius, F., \& Iglesias, I. (2013). Agronomical performance under Mediterranean climatic conditions among peach [Prunus persica (L.) Batsch] cultivars originated from different breeding programs. Scientia Horticulturae 150, 267-277. 
Reig, G., Iglesias, I., \& Echeverría, G. (2009). Agronomical performance, fruit quality and sensory attributes of several flat peach and flat nectarine cultivars. VII International Peach Symposium 962, 563-569.

Rinnan, Å., van den Berg, F., Engelsen, S.B. (2009). Review of the most common preprocessing techniques for near-infrared spectra. Trends in Analytical Chemistry 28, 12011222.

Serranti, S., Cesare, D., Marini, F. \& Bonifazi, G. (2013). Classification of oat and groat kernels using NIR hyperspectral imaging. Talanta 103, 276-284.

Sun, Y., Wang, Y., Xiao, Y., Gu, X., Pan, L. \& Tu, K. (2017). Hyperspectral imaging detection of decayed honey peaches based on their chlorophyll content. Food Chemistry 235, 194-202

USDA. (2016). EU-28 Stone Fruit Annual. https://gain.fas.usda.gov/ Accessed 15.04.17.

Valero, C., Crisosto, C. H. \& Slaughter, D. (2007). Relationship between nondestructive firmness measurements and commercially important ripening fruit stages for peaches, nectarines and plums. Postharvest Biology and Technology 44, 248-253.

Vélez-Rivera, N., Gómez-Sanchis, J., Chanona-Pérez, J.J., Carrasco, J.J., Millán-Giraldo, M., Lorente, D., Cubero, S. \& Blasco, J. (2014). Early detection of mechanical damage in mango using NIR hyperspectral images and machine learning. Biosystems Engineering 122, 91-98.

Verdú, S., Vásquez, F., Grau, R., Ivorra, E., Sanchez, A.J., \& Barat J.M. (2016). Detection of adulterations with different grains in wheat products based on the hyperspectral image technique: The specific cases of flour and bread. Food Control 62, 373-380.

Yang, C.H., Sun, D.W., Pu, H., Wang, N.N. \& Zhu, Z. (2015). Rapid detection of anthocyanin content in lychee pericarp during storage using hyperspectral imaging coupled with model fusion. Postharvest Biology and Technology 103, 55-65.

Zhang, B., Li, J., Fan, S., Huang, W., Zhao, C., Liu, C. \& Huang, D. (2015). Hyperspectral imaging combined with multivariate analysis and band math for detection of common defects on peaches (Prunus persica). Computers and Electronics in Agriculture 114, 14-24. 
611 Figure 1. Mean hyperspectral image spectra of 'Diamond Ray' and 'Big Top' cultivars

612

613 Figure 2. Score image of the two first PC of the PCA model using pixel spectra of 40 fruits of each cultivar from the calibration set

615 Key for Figure 2: The percentages indicate the explained variance (87.8\% of the total variance).

616 The variations in the colour in both score plots show features linked to the distribution or content of the biochemical constituents in each fruit and cultivar. 'DR'='Diamond Ray'; 'BT'= 'Big Top'

619

Figure 3. Score (A) and loadings plot (B) of the PCA of the mean spectra of the calibration set

622 Figure 4. Visualisation of cultivar classification using individual pixel spectrum: A) Pixel 623 classification method; B) Object classification method Key for Figure 4: Blue = 'Diamond Ray'; Red = 'Big Top'

625

Figure 5. Visualisation of cultivar classification using mean spectrum: A) Classification using the full range; B) Classification using 14 optimal wavelengths Key for Figure 5: Blue = 'Diamond Ray'; Red = 'Big Top'

630 Figure 6. Vector of regression coefficients of the PLS-DA model using mean spectra and with 631 the optimal wavelengths selected 